\title{
ASSOCIATION OF PARACOCCIDIOIDOMYCOSIS AND COVID-19 IN AN IMMUNOSUPPRESSED PATIENT WITH ANTISYNTHETASE SYNDROME - CASE REPORT
}

Laura Maria Silva de Siqueira1,*, Douglas Squizatto Leite ${ }^{1}$, João Flávio Gomes Faria ${ }^{1}$, Daniela Esteves Temporim¹, Andrea de Almeida Peduti Batista ${ }^{1}$, Henrique Pereira Sampaio ${ }^{1}$, Luiz Eduardo Valente ${ }^{1}$, Sula Glaucia Lage Drumond Pacheco ${ }^{1}$, Sean Hideo Shirata Lanças ${ }^{1}$, Matheus Zanata Brufatto ${ }^{1}$

1.Universidade Estadual Paulista “Júlio de Mesquita Filho", Botucatu (SP), Brazil.

*Corresponding author: mss.laura@yahoo.com

\section{BACKGROUND}

Paracoccidioidomycosis is a systemic mycosis caused by Paracoccidioides Brasiliensis and Lutzii, Brazil being the main endemic area in Latin America. Reports show an association with immunotherapy, such as anti-TNF. We are currently experiencing the greatest health crisis in the world due to the pandemic caused by COVID-19. Brazil is one of the main countries with the number of deaths and infections.

\section{CASE REPORT}

Male, 34 years old, with antisynthetase syndrome: proximal weakness of the pelvic and scapular girdle, ENMG with a myopathic pattern, CPK elevation: $12525 \mathrm{U} / \mathrm{L}$, mechanic's hands, Raynaud's phenomenon, dysphagia, asymmetric polyarthritis, cough, dyspnea with interstitial lung disease, positive Jo-1 antibody, ANA 1/320 cytoplasmic fine speckled pattern. Prednisone $1 \mathrm{mg} / \mathrm{kg} / \mathrm{day}$ and methotrexate $15 \mathrm{mg} /$ week were started for 1 year and switched to azathioprine $2 \mathrm{mg} / \mathrm{kg} /$ day due to worsening joint and pulmonary involvement. Recurrent of the joints, myopathic and pulmonary led to associate methotrexate and azathioprine, without reply. Pulse therapy with methylprednisolone $3 \mathrm{~g}$ and 12 pulses of Cyclophosphamide 0.6 to $1 \mathrm{~g} / \mathrm{m}^{2}$ were performed, with clinical improvement, but with the persistence of muscle enzymes. Started then rituximab $2 \mathrm{~g}$ ( $1 \mathrm{~g}$ followed by $1 \mathrm{~g}$ after 14 days) every six months (induction cycle), with normalization of CPK levels, strength, and reduction of ground-glass areas. After three induction cycles, it was decreased to $1 \mathrm{~g}$ every six months (maintaining cycle). After three maintaining cycles, he presented, for two months, asymmetric polyarthritis, wasting syndrome $(20 \mathrm{~kg}$ ), night fever, cough without CPK elevation, and waist weakness. Tomography showing multiple, bilateral, multilobar ground-glass opacities (Figure 1). Because of the pulmonary condition, collected nasal swab (RT-PCR) with a positive COVID-19 result. Further investigation, tuberculin test, bacilloscopy and culture of bronchoalveolar lavage (BAL) for Mycobacterium tuberculosis result negative. However, direct mycological test of BAL identified the presence of Paracoccidioides brasiliensis. Sulfamethoxazole trimethoprim 400/80 mg was started for 30 days and itraconazole $200 \mathrm{mg} /$ day. Complete resolution of symptoms and improvement in tomographic image after three months of treatment (Figure 2).

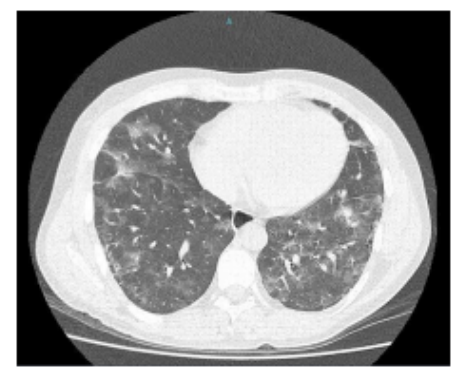

Figure 1. Ground-glass opacities.

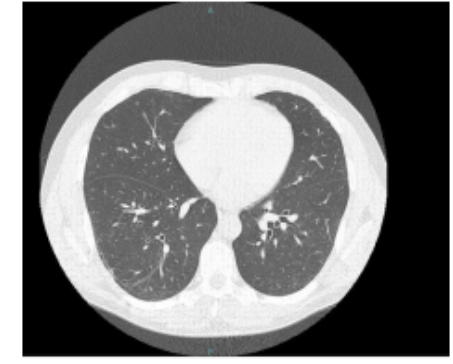

Figure 2. After three months of treatment.

\section{CONCLUSION}

In rheumatology clinical practice, infections may be associated with underlying disease triggers. In this case, the high degree of immunosuppression associated with an isolated subacute pulmonary condition, without recurrence of the myopathic condition, was essential for the suspicion of infection. The pandemic and endemic Brazilian epidemiological context were essential for diagnostic investigation and therapeutic success. Hospitalization or oxygen therapy wasn't necessary.

\section{KEYWORDS}

Paracoccidioidomycosis, COVID-19, Antisynthetase syndrome. 